\title{
WAVELET-BASED DISCRETE-CONTINUAL FINITE ELEMENT PLATE ANALYSIS WITH THE USE OF DAUBECHIES SCALING FUNCTIONS
}

\author{
Marina L. Mozgaleva ${ }^{1}$, Pavel A.Akimov ${ }^{2,3,4,5}$, Taymuraz B. Kaytukov ${ }^{2,6}$ \\ ${ }^{1}$ National Research Moscow State University of Civil Engineering, Moscow, RUSSIA \\ ${ }^{2}$ Russian Academy of Architecture and Building Sciences, Moscow, RUSSIA \\ ${ }^{3}$ Scientific Research Center "StaDyO", Moscow, RUSSIA \\ ${ }^{4}$ Tomsk State University of Architecture and Civil Engineering, Tomsk, RUSSIA \\ ${ }^{5}$ Peoples' Friendship University of Russia, Moscow, RUSSIA \\ ${ }^{6}$ Central Institute for Research and Design of the Ministry of Construction and Housing and Communal Services \\ of the Russian Federation, Moscow, RUSSIA
}

\begin{abstract}
The distinctive paper is detoded to special version of wavelet-based discrete-continual finite element method of plate analysis. Daubechies scaling functions are used within this version. Its field of application comprises plates with constant (generally piecewise constant) physical and geometrical parameters along one direction (so-called "basic" direction). Modified continual operational formulation of the problem with the use of the method of extended domain (proposed by A.B. Zolotov) is presented. Corresponding discrete-continual formulation is given as well. Brief information about computer implementation of the method with the use of MATLAB software is provided. Besides numerical sample of analysis of thin plate is considered.
\end{abstract}

Keywords: boundary problem, structural analysis, plate analysis, thin plate, numerical solution, wavelet-based discrete-continual finite element method, wavelet analysis,

Daubechies scaling function, Daubechies wavelet

\section{ВЕЙВЛЕТ-РЕАЛИЗАЦИЯ ДИСКРЕТНО-КОНТИНУАЛЬНОГО МЕТОДА КОНЕЧНЫХ ЭЛЕМЕНТОВ ДЛЯ РАСЧЕТА ПЛИТ С ИСПОЛЬЗОВАНИЕМ МАСШТАБИРУЮЩИХ ФУНКЦИЙ ДОБЕШИ}

\author{
М.Л. Мозгалева ${ }^{1}$, П.А. Акимов ${ }^{2,3,4,5}$, Т.Б. Кайтуков ${ }^{2,6}$ \\ ${ }^{1}$ Национальный исследовательский Московский государственный строительный университет, \\ г. Москва, РОССИЯ \\ ${ }^{2}$ Российская академия архитектуры и строительных наук, г. Москва, РОССИЯ \\ ${ }^{3}$ Научно-исследовательский центр СтаДиО, г. Москва, РОССИЯ \\ ${ }^{4}$ Томский государственный архитектурно-строительный университет, г. Томск, РОССИЯ \\ 5 Российский университет дружбы народов, г. Москва, РОССИЯ \\ ${ }^{6}$ Центральный научно-исследовательский и проектный институт \\ Министерства строительства и жилищно-коммунального хозяйства, г. Москва, РОССИЯ
}

\begin{abstract}
Аннотация: Настоящая статья посвящена одной специальной версии вейвлет-реализации дискретноконтинуального метода конечных элементов для расчета плитных конструкций. В рамках указанной версии используются масштабирующие функции Добеши. Область применения данного метода составляют пластины с постоянными (в общем случае кусочно-постоянными) физико-геометрическими параметрами по одному из направлений (это так называемое «основное» направление). В статье приведена преобразованная континуальная постановка задачи с использованием аппарата метода расширенной области, предложенного А.Б. Золотовым. Кроме того, здесь представлены соответствующая дискретно-контиунальная постановка, краткие сведения о компьютерной реализации метода с использованием системы MATLAB, а также пример расчета.
\end{abstract}


Ключевые слова: краевая задача, расчеты строительных конструкций, расчет пластин, тонкая пластина, численное решение, вейвлет-реализация дискретно-континуального метода конечных элементов, вейвлет-анализ, масштабирующая функция Добеши, вейвлет Добеши

As is known wavelet analysis has the desirable advantages of multi-resolution properties and various basis functions, which fulfill an enormous potential fo rsolving partial differential equations. The distinctive paper is devoted to further development of wavelet-based discretecontinual finite element method of structural analysis. Particularly problems of plate analysis with the use of Daubechies scaling functions [129] are under consideration.

\section{CONTINUAL FORMULATION OF PROBLEM}

Let $x_{1}, x_{2}$ are cartesian coordinates. Besides, let $x_{2}$ be coordimate corresponding to "basic" direction of plate (i.e. direction along which physical and geometrical parameters of plate are constant). It is is necessary to note that physical and geometrical parameters of plate can be changed arbitrarily along $x_{1}$. Let us consider the following domain occupied by plate:

$$
\Omega=\left\{\left(x_{1}, x_{1}\right): 0<x_{1}<\ell_{1}, 0<x_{2}<\ell_{2}\right\} .
$$

Operational formulation of coorresponding boundary problem of plate analysis (Kirghoff model) at extended domain [30], embordering considering structure has the form:

$$
L y=\tilde{F}, \quad 0 \leq x_{1} \leq \ell_{1}, \quad 0 \leq x_{2} \leq \ell_{2},
$$

where $y$ is plate deflection in domain $\Omega ; L$ is the operator of the considering problem; $\tilde{F}$ is the corresponding right-side function;

$$
\begin{gathered}
L=-L_{4} \partial_{2}^{4}+L_{2} \partial_{2}^{2}+L_{0} \\
L_{4}=\theta D \\
L_{2}=-\left[\partial_{1}^{2} \theta D v+2 \partial_{1} D(1-v) \partial_{1}+\theta D v \partial_{1}^{2}\right] \\
L_{0}=-\partial_{1}^{2} \theta D \partial_{1}^{2}
\end{gathered}
$$

$$
\begin{gathered}
\tilde{F}=\theta F+\delta_{\Gamma} f ; \\
\partial_{j}=\partial / \partial x_{j}, \quad \partial_{j}^{*}=-\partial / \partial x_{j}, \quad j=1,2 ;
\end{gathered}
$$

$\bar{F}$ is the force in domain $\Omega ; \bar{f}_{k}$ is the boundary force at $\Gamma=\partial \Omega ; D, v$ are plate modulus and Poison's ratio in domain $\Omega$;

$$
D=E h^{3} /\left[12\left(1-v^{2}\right)\right]
$$

$h$ is plate thickness in domain $\Omega$;

$$
\theta=\theta\left(x_{1}, x_{2}\right)= \begin{cases}1, & \left(x_{1}, x_{2}\right) \in \Omega \\ 0, & \left(x_{1}, x_{2}\right) \notin \Omega ;\end{cases}
$$

is the characteristic function of domain $\Omega$ [30];

$$
\delta_{\Gamma}=\delta_{\Gamma}\left(x_{1}, x_{2}\right)=\frac{\partial \theta}{\partial \bar{n}} ;
$$

is the delta-function of border $\Gamma=\partial \Omega_{k}$; $\bar{n}=\left[\begin{array}{ll}n_{1} & n_{2}\end{array}\right]^{T}$ is unit normal vector of domain boundary $\Gamma=\partial \Omega[30]$;

Let us introduce the following notation [4,7-15]:

$$
\begin{aligned}
& y_{1}=y, \quad y_{2}=\partial_{2} y=y_{1}^{\prime}, \\
& y_{3}=\partial_{2}^{2} y=y_{2}^{\prime}, \quad y_{4}=\partial_{2}^{3} y=y_{3}^{\prime} .
\end{aligned}
$$

Therefor we can rewrite (1.2) in the form

$$
-L_{4} y_{4}^{\prime}+L_{2} y_{3}+L_{0} y_{1}=\tilde{F} \text {. }
$$

Taking into account (1.12) and (1.13), we get

$$
\left.\left\lfloor\begin{array}{l}
y_{1}^{\prime} \\
y_{2}^{\prime} \\
y_{3}^{\prime} \\
y_{4}^{\prime}
\end{array}\right\rfloor=\left[\begin{array}{cccc}
0 & 1 & 0 & 0 \\
0 & 0 & 1 & 0 \\
0 & 0 & 0 & 1 \\
L_{4}^{-1} L_{0} & 0 & L_{4}^{-1} L_{2} & 0
\end{array}\right\rfloor \| \begin{array}{l}
y_{1} \\
y_{2} \\
y_{3} \\
y_{4}
\end{array}\right\rfloor=\left\lfloor\begin{array}{c}
0 \\
0 \\
0 \\
-L_{4}^{-1} \tilde{F}
\end{array}\right\rfloor .
$$




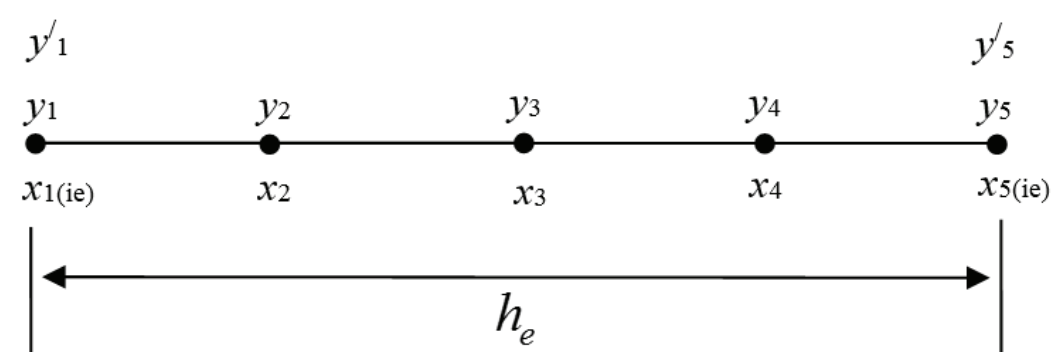

Figure 1. Approximation on the element.

We can also rewrite (1.14) in matrix form

$$
\bar{U}^{\prime}=\tilde{L} \bar{U}+\overline{\widetilde{F}},
$$

where

$$
\begin{aligned}
& \tilde{L}=\left\lfloor\begin{array}{cccc}
0 & 1 & 0 & 0 \\
0 & 0 & 1 & 0 \\
0 & 0 & 0 & 1 \\
L_{4}^{-1} L_{0} & 0 & L_{4}^{-1} L_{2} & 0
\end{array}\right\rfloor ; \\
& \overline{\widetilde{F}}=\left\lfloor\begin{array}{c}
0 \\
0 \\
0 \\
-L_{4}^{-1} \tilde{F}
\end{array}\right] ; \quad \bar{U}=\left\lfloor\begin{array}{l}
y_{1} \\
y_{2} \\
y_{3} \\
y_{4}
\end{array}\right\rfloor ;
\end{aligned}
$$

Besides, the system of equations (1.15) is supplemented by the boundary conditions that are specified in sections with coordinates (coordinates of boundary points)

$$
x_{2,1}^{b}=0, \quad x_{2,2}^{b}=\ell_{2} .
$$

\section{DISCRETE-CONTINUAL FORMULATION OF PROBLEM}

The discrete component of the numerical solution is represented by the direction along the axis $x_{1}$. Fulfillment on an element (interval) for all components of the vector-function $\bar{U}$ is the same. Therefore, for simplicity let us denote locally in this paragraph

$$
x=x_{1}, \quad \ell=\ell_{1}, \quad y=y_{j}
$$

for all $j=1,2,3,4$. We can divide the interval $[0, \ell]$ into $N_{e}$ parts (elements) and $h_{e}=\ell / N_{e}$ is the length of each element. Besides, we can divide each element into $N_{k}$ parts (for instance, sample with $N_{k}=4$ is presented in Fig. 1. Let us use the following notation: $i_{e}$ is the number of the element; $x_{1}\left(i_{e}\right)$ is the coordinate of the starting point; $x_{5}\left(i_{e}\right)$ is the coordinate of the end point of the ith element. Let $y_{i}$ and $y_{i}^{\prime}=\partial_{1} y\left(x_{i}\right)$ be unknowns at boundary points $(i=1,5)$. Let $y_{i}$ be unknown internal points $(i=2,3,4)$. Thus, the total number of unknowns on an element with such discretization is equal to $N=N_{k}-1+2 \cdot 2=N_{k}+3=7$.

Let us introduce the local coordinate within the element:

$$
t=\frac{x-x_{1\left(i_{e}\right)}}{h_{e}}, \quad x_{1\left(i_{e}\right)} \leq x \leq x_{5\left(i_{e}\right)} .
$$

Thus, we have the following formulas:

$$
\begin{aligned}
& \left\{\begin{array}{l}
x=x_{1\left(i_{e}\right)} \Rightarrow t=0 \\
x=x_{2} \Rightarrow t=0.25 \\
x=x_{3} \Rightarrow t=0.5 \\
x=x_{4} \Rightarrow t=0.75 \\
x=x_{5\left(i_{e}\right)} \Rightarrow t=1 ;
\end{array}\right. \\
& \frac{d}{d x}=\frac{d}{d t} \cdot \frac{d t}{d x}=\frac{1}{h_{e}} \frac{d}{d t} ; \quad \frac{d^{p}}{d x^{p}}=\frac{1}{h_{e}^{p}} \frac{d^{p}}{d t^{p}} ;
\end{aligned}
$$


Wavelet-Based Discrete-Continual Finite Element Plate Analysis with the Use of Daubechies Scaling Functions

$$
d x=h_{e} \cdot d t
$$

In order to construct local stiffness matrices corresponding to continual operators (1.6), (1.5) and (1.4) let us consider bilinear forms taking into account relations (2.3)-(2.5):

$$
\begin{aligned}
B_{0}(y, z)= & <L_{0} y, z>= \\
= & -\int_{x_{1\left(i_{e}\right)}}^{x_{5\left(i_{e}\right)}} \frac{d^{2}}{d x^{2}} \theta D \frac{d^{2} y}{d x^{2}} \cdot z d x= \\
= & -\theta_{i_{e}} D_{i_{e}} \int_{x_{1\left(i_{e}\right)}}^{x_{5\left(i_{e}\right)}} \frac{d^{2} y}{d x^{2}} \cdot \frac{d^{2} z}{d x^{2}} d x= \\
= & -\frac{1}{h_{e}^{3}} \theta_{i_{e}} D_{i_{e}} \int_{0}^{1} \frac{d^{2} w}{d t^{2}} \cdot \frac{d^{2} v}{d t^{2}} d t= \\
= & B_{0}(w, v) ; \\
B_{4}(y, z) & =<L_{4} y, z>= \\
& =\theta_{i_{e}} D_{i_{e}} \int_{x_{1\left(i_{e}\right)}}^{x_{5\left(i_{e}\right)}} y \cdot z d x= \\
& =h_{e} \theta_{i_{e}} D_{i_{e}} \int_{0}^{1} w \cdot v d t=B_{4}(w, v) \\
B_{2}(y, z) & =<L_{2} y, z>= \\
& =<L_{21} y, z>+<L_{22} y, z>+ \\
& +<L_{23} y, z>
\end{aligned}
$$

where

$$
\begin{aligned}
<L_{21} y, z> & =-\theta_{i_{e}} D_{i_{e}} v_{i_{e}} \int_{x_{1\left(i_{e}\right)}}^{x_{5\left(i_{e}\right)}} \frac{d^{2} y}{d x^{2}} z d x= \\
& =-\frac{1}{h_{e}} \theta_{i_{e}} D_{i_{e}} v_{i_{e}} \int_{0}^{1} \frac{d^{2} w}{d t^{2}} \cdot v d t= \\
& =B_{21}(w, v) ; \\
<L_{23} y, z>= & -\theta_{i_{e}} D_{i_{e}} v_{i_{e}} \int_{x_{1\left(i_{e}\right)}}^{x_{5\left(i_{e}\right)}} y \cdot \frac{d^{2} z}{d x^{2}} d x= \\
= & -\frac{1}{h_{e}} \theta_{i_{e}} D_{i_{e}} v_{i_{e}} \int_{0}^{1} w \cdot \frac{d^{2} v}{d t^{2}} d t= \\
= & B_{23}(w, v) ;
\end{aligned}
$$

$$
\begin{aligned}
<L_{22} y, z> & =-2 \int_{x_{1\left(i_{e}\right)}}^{x_{5\left(i_{e}\right)}} \frac{d}{d x} \theta D(1-v) \frac{d y}{d x} \cdot z d x= \\
= & 2 \theta_{i_{e}} D_{i_{e}}\left(1-v_{i_{e}}\right) \int_{x_{1\left(i_{e}\right)}}^{x_{5\left(i_{e}\right)}} \frac{d y}{d x} \cdot \frac{d z}{d x} d x= \\
= & \frac{1}{h_{e}} 2 \theta_{i_{e}} D_{i_{e}}\left(1-v_{i_{e}}\right) \int_{0}^{1} \frac{d w}{d t} \cdot \frac{d v}{d t} d t= \\
= & B_{22}(w, v) \\
y(x)=w(t)= & \sum_{k=0}^{N-1} \alpha_{k} \varphi(t+k), \\
& x_{1\left(i_{e}\right)} \leq x \leq x_{5\left(i_{e}\right)}, \quad 0 \leq t \leq 1 ; \\
z(x)=v(t)= & \sum_{k=0}^{N-1} \beta_{k} \varphi(t+k), \quad \\
& x_{1\left(i_{e}\right)} \leq x \leq x_{5\left(i_{e}\right)}, \quad 0 \leq t \leq 1 ;
\end{aligned}
$$

$\varphi(s)$ is Daubechies scaling function,

$$
[0, N] \subseteq \sup \operatorname{p} \varphi
$$

Thus, we can substitute (2.12), (2.13) sequentially in (2.6)-(2.11):

$$
\begin{aligned}
& B_{0}(w, v)=-\frac{1}{h_{e}^{3}} \theta_{i_{e}} D_{i_{e}} \int_{0}^{1} \frac{d^{2} w}{d t^{2}} \cdot \frac{d^{2} v}{d t^{2}} d t= \\
& -\frac{\theta_{i_{e}} D_{i_{e}}}{h_{e}^{3}} \sum_{i=0}^{N-1} \sum_{j=0}^{N-1} \alpha_{i} \beta_{j} \int_{0}^{1} \varphi^{\prime \prime}(t+i) \varphi^{\prime \prime}(t+j) d t= \\
& =-\frac{\theta_{i_{e}} D_{i_{e}}}{h_{e}^{3}}\left(K_{\alpha \beta}^{0} \bar{\alpha}, \bar{\beta}\right),
\end{aligned}
$$

where

$$
K_{\alpha \beta}^{0}(i, j)=\int_{0}^{1} \varphi^{\prime \prime}(t+i) \varphi^{\prime \prime}(t+j) d t, \quad \varphi^{\prime \prime}=\frac{d^{2} \varphi}{d t^{2}}
$$

$$
\begin{aligned}
& B_{4}(w, v)=h_{e} \theta_{i_{e}} D_{i_{e}} \int_{0}^{1} w \cdot v d t= \\
& =\theta_{i_{e}} D_{i_{e}} h_{e} \sum_{i=0}^{N-1} \sum_{j=0}^{N-1} \alpha_{i} \beta_{j} \int_{0}^{1} \varphi(t+i) \varphi(t+j) d t= \\
& =h_{e} \theta_{i_{e}} D_{i_{e}}\left(K_{\alpha \beta}^{4} \bar{\alpha}, \bar{\beta}\right) ;
\end{aligned}
$$




$$
\begin{aligned}
\theta_{i_{e}} D_{i_{e}} h_{e} \sum_{i=0}^{N-1} \sum_{j=0}^{N-1} \alpha_{i} \beta_{j} \int_{0}^{1} \varphi(t+i) \varphi(t+j) d t= \\
=h_{e} \theta_{i_{e}} D_{i_{e}}\left(K_{\alpha \beta}^{4} \bar{\alpha}, \bar{\beta}\right)
\end{aligned}
$$

where

$$
\begin{aligned}
& K_{\alpha \beta}^{4}(i, j)=K_{\alpha \beta}^{4}(i, j)=\int_{0}^{1} \varphi(t+i) \varphi(t+j) d t ; \\
& B_{21}(w, v)=-\frac{1}{h_{e}} \theta_{i_{e}} D_{i_{e}} v_{i_{e}} \int_{0}^{1} \frac{d^{2} w}{d t^{2}} \cdot v d t= \\
& =-\frac{\theta_{i_{e}} D_{i_{e}} v_{i_{e}}}{h_{e}} \sum_{i=0}^{N-1} \sum_{j=0}^{N-1} \alpha_{i} \beta_{j} \int_{0}^{1} \varphi^{\prime \prime}(t+i) \varphi(t+j) d t= \\
& =-\frac{\theta_{i_{e}} D_{i_{e}} v_{i_{e}}}{h_{e}}\left(K_{\alpha \beta}^{21} \bar{\alpha}, \bar{\beta}\right),
\end{aligned}
$$

where

$$
\begin{gathered}
K_{\alpha \beta}^{21}(i, j)=\int_{0}^{1} \varphi^{\prime \prime}(t+i) \varphi(t+j) d t ; \\
B_{23}(w, v)=-\frac{1}{h_{e}} \theta_{i_{e}} D_{i_{e}} v_{i_{e}} \int_{0}^{1} w \cdot \frac{d^{2} v}{d t^{2}} d t= \\
=-\frac{\theta_{i_{e}} D_{i_{e}} v_{i_{e}}}{h_{e}} \sum_{i=0}^{N-1} \sum_{j=0}^{N-1} \alpha_{i} \beta_{j} \int_{0}^{1} \varphi(t+i) \varphi^{\prime \prime}(t+j) d t= \\
=-\frac{\theta_{i_{e}} D_{i_{e}} v_{i_{e}}}{h_{e}}\left(K_{\alpha \beta}^{23} \bar{\alpha}, \bar{\beta}\right),
\end{gathered}
$$

where

$$
K_{\alpha \beta}^{23}(i, j)=\int_{0}^{1} \varphi(t+i) \varphi^{\prime \prime}(t+j) d t=K_{\alpha \beta}^{21}(j, i) ;
$$

where

$$
\begin{aligned}
\bar{y}^{i_{e}} & =\left[\begin{array}{lllllll}
y_{1} & \frac{d y_{1}}{d x} & y_{2} & y_{3} & y_{4} & y_{5} & \frac{d y_{5}}{d x}
\end{array}\right]^{T} \\
\bar{\alpha} & =\left[\begin{array}{lllllll}
\alpha_{0} & \alpha_{1} & \alpha_{2} & \alpha_{3} & \alpha_{4} & \alpha_{5} & \alpha_{6}
\end{array}\right]^{T}
\end{aligned}
$$

We define the parameters $\alpha_{k}$ and $\beta_{k}$ through the node unknowns on the element:

$$
\left\{\begin{array}{l}
y_{1}=w(0)=\sum_{k=0}^{N-1} \alpha_{k} \varphi(k) \\
\frac{d y_{1}}{d x}=\frac{1}{h_{e}} w^{\prime}(0)=\frac{1}{h_{e}} \sum_{k=0}^{N-1} \alpha_{k} \varphi^{\prime}(k) \\
y_{2}=w(0.25)=\sum_{k=0}^{N-1} \alpha_{k} \varphi(k+0.25) \\
y_{3}=w(0.5)=\sum_{k=0}^{N-1} \alpha_{k} \varphi(k+0.5) \\
y_{4}=w(0.75)=\sum_{k=0}^{N-1} \alpha_{k} \varphi(k+0.75) \\
y_{5}=w(1)=\sum_{k=0}^{N-1} \alpha_{k} \varphi(k+1) \\
\frac{d y_{5}}{d x}=\frac{1}{h_{e}} w^{\prime}(1)=\frac{1}{h_{e}} \sum_{k=0}^{N-1} \alpha_{k} \varphi^{\prime}(k+1)
\end{array}\right.
$$

Therefor we have

$$
\bar{y}^{i_{e}}=T \bar{\alpha},
$$


Wavelet-Based Discrete-Continual Finite Element Plate Analysis with the Use of Daubechies Scaling Functions

$$
\left.T=D \mid \begin{array}{ccccccc}
\varphi(0) & \varphi(1) & \varphi(2) & \varphi(3) & \varphi(4) & \varphi(5) & \varphi(6) \\
\varphi^{\prime}(0) & \varphi^{\prime}(1) & \varphi^{\prime}(2) & \varphi^{\prime}(3) & \varphi^{\prime}(4) & \varphi^{\prime}(5) & \varphi^{\prime}(6) \\
\varphi(0.25) & \varphi(1.25) & \varphi(2.25) & \varphi(3.25) & \varphi(4.25) & \varphi(5.25) & \varphi(6.25) \\
\varphi(0.5) & \varphi(1.5) & \varphi(2.5) & \varphi(3.5) & \varphi(4.5) & \varphi(5.5) & \varphi(6.5) \\
\varphi(0.75) & \varphi(1.75) & \varphi(2.75) & \varphi(3.75) & \varphi(4.75) & \varphi(5.75) & \varphi(6.75) \\
\varphi(1) & \varphi(2) & \varphi(3) & \varphi(4) & \varphi(5) & \varphi(6) & \varphi(7) \\
\varphi^{\prime}(1) & \varphi^{\prime}(2) & \varphi^{\prime}(3) & \varphi^{\prime}(4) & \varphi^{\prime}(5) & \varphi^{\prime}(6) & \varphi^{\prime}(7)
\end{array}\right]
$$

Similarly, we get

$$
L_{2}=L_{21}+L_{22}+L_{23},
$$

$$
\bar{z}^{i_{e}}=T \bar{\beta}
$$

Taking into account (2.26) and (2.31) we get

we have the following local stiffness matrix

$$
K_{2}^{i_{e}}=K_{21}^{i_{e}}+K_{22}^{i_{e}}+K_{23}^{i_{e}}
$$

$$
\bar{\alpha}=T^{-1} \bar{y}^{i_{e}} ; \quad \bar{\beta}=T^{-1} \bar{z}^{i_{e}} .
$$

In the general case, the following chain of equalities holds:

$$
\begin{aligned}
& \left(K_{\alpha \beta} \bar{\alpha}, \bar{\beta}\right)=\left(K_{\alpha \beta} T^{-1} \bar{y}^{i_{e}}, T^{-1} \bar{z}^{i_{e}}\right)= \\
& \quad=\left(\left(T^{-1}\right)^{\mathrm{T}} K_{\alpha \beta} T^{-1} \bar{y}^{i_{e}}, \bar{z}^{i_{e}}\right) .
\end{aligned}
$$

Therefore, substituting (2.32) sequentially into (2.14), (2.16), (2.19), (2.21), (2.23), we obtain local stiffness matrices $K_{0}^{i_{e}}, K_{4}^{i_{e}}, K_{21}^{i_{e}}, K_{23}^{i_{e}}$, $K_{22}^{i_{e}}, K_{2}^{i_{e}}$, corresponding to the operators $L_{0}$, $L_{4}, L_{21}, L_{23}, L_{22}, L_{2}$,

$$
\begin{gathered}
K_{0}^{i_{e}}=-\frac{\theta_{i_{e}} D_{i_{e}}}{h_{e}^{3}}\left(T^{-1}\right)^{\mathrm{T}} K_{\alpha \beta}^{0} T^{-1} ; \\
K_{4}^{i_{e}}=h_{e} \theta_{i_{e}} D_{i_{e}}\left(T^{-1}\right)^{\mathrm{T}} K_{\alpha \beta}^{4} T^{-1} ; \\
K_{21}^{i_{e}}=-\frac{\theta_{i_{e}} D_{i_{e}} v_{i_{e}}}{h_{e}}\left(T^{-1}\right)^{\mathrm{T}} K_{\alpha \beta}^{21} T^{-1} ; \\
K_{23}^{i_{e}}=-\frac{\theta_{i_{e}} D_{i_{e}} v_{i_{e}}}{h_{e}}\left(T^{-1}\right)^{\mathrm{T}} K_{\alpha \beta}^{23} T^{-1}=\left(K_{21}^{i_{e}}\right)^{T} ; \\
K_{22}^{i_{e}}=2 \frac{\theta_{i_{e}} D_{i_{e}}\left(1-v_{i_{e}}\right)}{h_{e}}\left(T^{-1}\right)^{\mathrm{T}} K_{\alpha \beta}^{22} T^{-1}
\end{gathered}
$$

Due to the fact that

where

$$
\begin{gathered}
\varphi^{\prime}\left(t_{k}\right) \approx d \varphi_{k}=\frac{\varphi_{k+1}-\varphi_{k-1}}{2 h_{t}}, \quad k=1,2, \ldots, N_{l} ; \\
\varphi^{\prime \prime}\left(t_{k}\right) \approx d 2 \varphi_{k}=\frac{\varphi_{k+1}-2 \varphi_{k}+\varphi_{k-1}}{h_{t}^{2}}, \\
\quad k=1,2, \ldots, N_{l},
\end{gathered}
$$




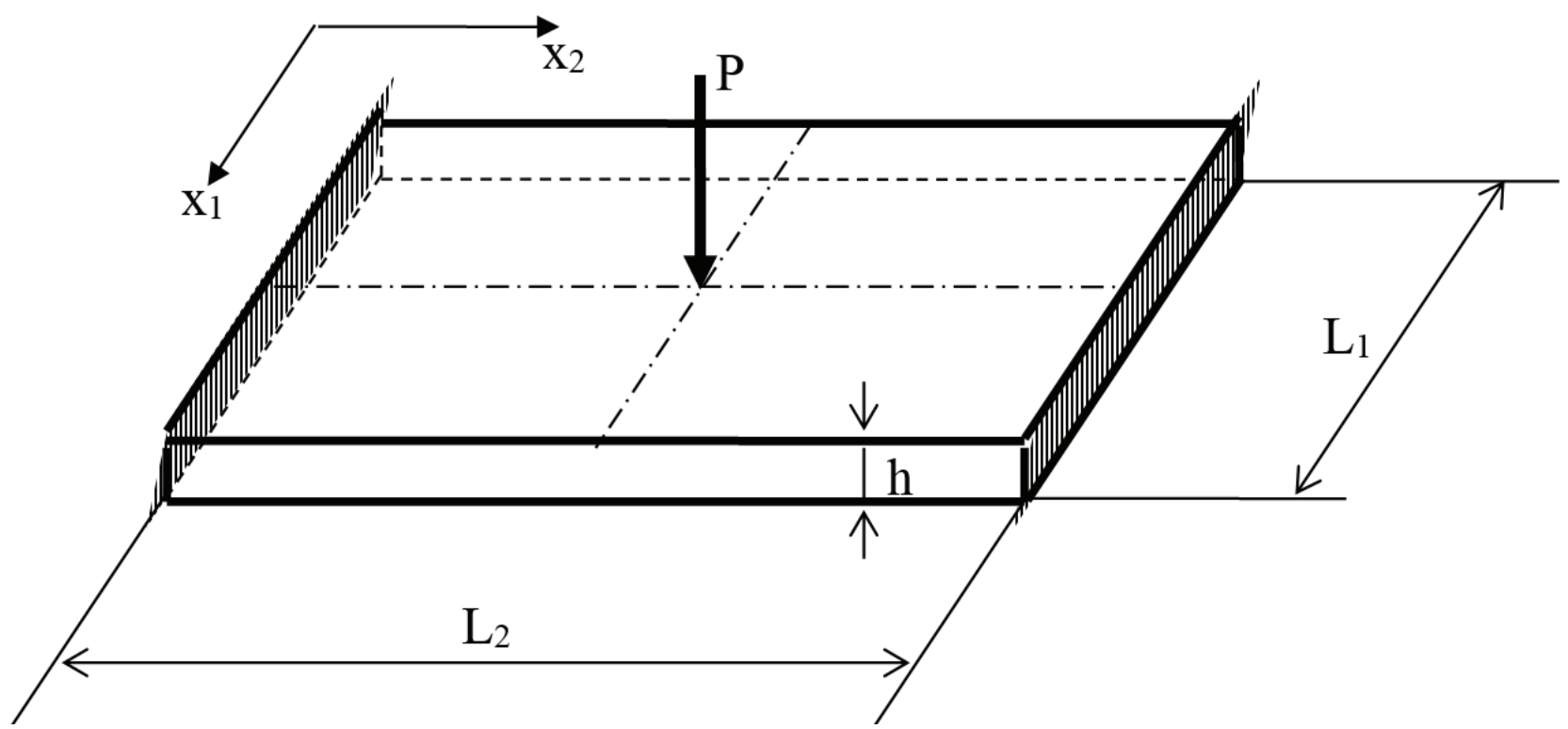

Figure 2. About formulation of the problem.

If $t_{k} \notin[0,27]$ then $\varphi_{k}=\varphi\left(t_{k}\right)=0$.

When computing the coefficients of the local stiffness matrix (formulas (2.14), (2.16), (2.19), (2.21), (2.23)), one can use the simplest quadrature formulas for numerical integration, in particular, midpoint quadrature rule with step $2 h_{t}$.

As an example, we consider the problem of bending a thin plate rigidly fixed along the lateral faces under the influence of a load concentrated in the center (Figure 2).

Geometric parameters: $L_{1}=0.9 \mathrm{~m}, L_{2}=1.0 \mathrm{~m}$, $h=0.05 \mathrm{~m}$ (thickness). The calculated parameters of the plate material: coefficient of elasticity $E=3000 \cdot 10^{4} \mathrm{kN} / \mathrm{m}^{2}, v=0.16$ is Poisson's ratio. External load parameter: $P=1 \mathrm{kN}$.

Let $N_{e}=3$ be the number of elements.

The length of the element

$$
h_{e}=L_{1} / N_{e}=0.9 / 3=0.3 \text {. }
$$

Distance between coordinates of nodes

$$
h_{p}=h_{e} / 4=0.3 / 4=0.075 .
$$

The number of nodal unknowns for each component of the vector function $y_{j}, j=1,2,3,4$ :

$$
N_{g}=N_{p}+2 N_{b}=3 \cdot 3+2 \cdot(3+1)=17,
$$

where $N_{p}=N_{e}\left(N_{k}-1\right)$ is the total number of internal nodes for all elements; $N_{b}=N_{e}+1$ is the total number of boundary nodes for all elements. Total number of unknowns is equal to

$$
N_{U}=4 N_{g}=4 \cdot 17=68
$$

For comparison, we use the traditional finite element method, where unknown functions on an element are represented as a cubic parabola and in the node each unknown function is represented by two unknown nodal quantities: the nodal value of the unknown function itself and its first derivative in the discrete direction.

The total number of nodal points in a discrete direction $x_{1}$ is equal to

$$
N_{1}=L_{1} / h_{p}+1=0.9 / 0.075+1=13 .
$$

The number of nodal unknowns for each component of the vector function $y_{j}, j=1,2,3,4$ :

$$
N_{g}=2 N_{1}=26
$$

Total number of unknowns is equal to 


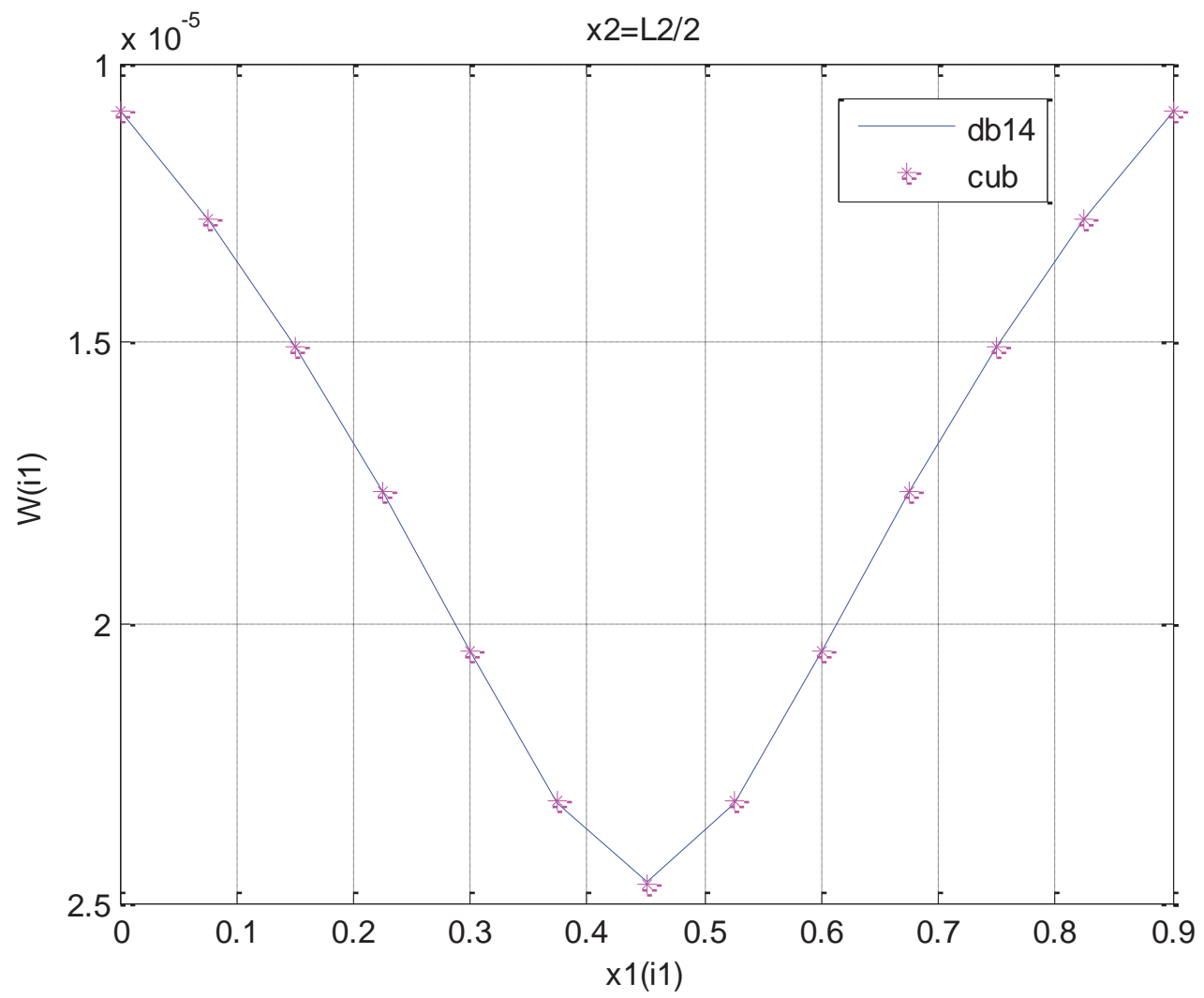

a)

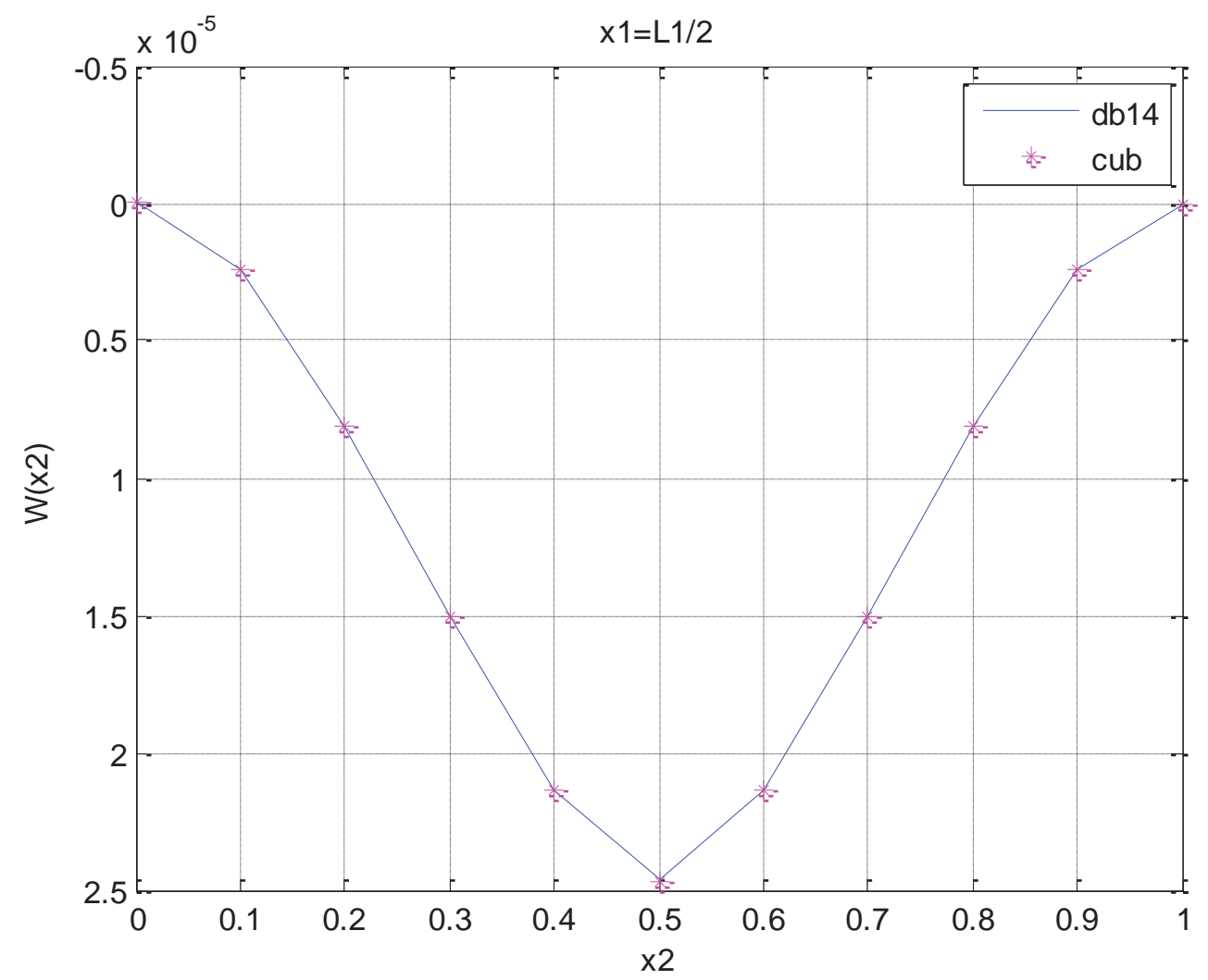

b)

Figure 3. Comparison of results in mid sections in each direction. 


$$
N_{U}=4 N_{g}=4 \cdot 26=104 .
$$

Graphical comparison of the results of analysis is presented at Figure 3 (db14 is deflection values obtained using the Daubechies scaling function; cub is deflection values obtained, obtained on the basis of the traditional finite element method; $h_{1}=0.075, h_{2}=0.1$ are steps of issuing results in the directions $x_{1}$ and $x_{2}$, respectively.

As can be seen, the results obtained practically coincide. Moreover, the finite element method algorithm based on the Daubechies scaling function leads to a significant decrease in the number of unknowns. The difference is equal to $4 N_{p}$.

\section{REFERENCES}

1. Daubechies I. Orthonormal bases of compactly supported wavelets. // Commun. Pure Appl. Math., 1988, vol. 41, pp. 909-996.

2. Akimov P.A., Aslami M. About Verification of Correct Wavelet-Based Approach to Local Static Analysis of Bernoulli Beam. // Applied Mechanics and Materials, 2014, Vols. 580-583, pp. 3013-3016.

3. Akimov P.A., Aslami M. Theoretical Foundations of Correct Wavelet-Based Approach to Local Static Analysis of Bernoulli Beam. // Applied Mechanics and Materials, 2014, Vols. 580-583, pp. 2924-2927.

4. Akimov P.A., Belostotsky A.M., Kaytukov T.B., Mozgaleva M.L., Aslami M. About several numerical and semianalytical methods of local structural analysis. // International Journal for Computational Civil and Structural Engineering, 2018, Vol. 14, Issue 4, pp. 59-69.

5. Akimov P.A., Mozgaleva M.L. Correct Wavelet-based Multilevel Numerical Method of Local Solution of Boundary Problems of Structural Analysis. // Applied Mechanics and Materials, 2012, Vols. 166-169, pp. 3155-3158.
6. Aslami M., Akimov P.A. Wavelet-based finite element method for multilevel local plate analysis. // Thin-Walled Structures, 2015-2016, Vol. 98, Part B, pp. 392-402.

7. Akimov P.A., Belostotsky A.M., Mozgaleva M.L., Aslami M., Negrozov O.A. Correct Multilevel Discrete-Continual Finite Element Method of Structural Analysis. // Advanced Materials Research, 2014, Vol. 1040, pp. 664-669.

8. Akimov P.A., Belostotsky A.M., Sidorov V.N., Mozgaleva M.L., Negrozov O.A. Application of discrete-continual finite element method for global and local analysis of multilevel systems. // Applied Mechanics and Materials; AIP Conference Proceedings, 2014, Vol. 1623(3), pp. 3-6.

9. Akimov P.A., Mozgaleva M.L. Correct Wavelet-based Multilevel DiscreteContinual Methods for Local Solution of Boundary Problems of Structural Analysis. // Applied Mechanics and Materials, 2013, Vols. 353-356, pp. 3224-3227.

10. Akimov P.A., Mozgaleva M.L. Waveletbased Multilevel Discrete-Continual Finite Element Method for Local Deep Beam Analysis. // Applied Mechanics and Materials, 2013, Vols. 405-408, pp. 3165-3168.

11. Akimov P.A., Mozgaleva M.L. Waveletbased Multilevel Discrete-Continual Finite Element Method for Local Plate Analysis. // Applied Mechanics and Materials, 2013, Vols. 351-352, pp. 13-16.

12. Akimov P.A., Mozgaleva M.L., Aslami M., Negrozov O.A. Modified Waveletbased Multilevel Discrete-Continual Finite Element. Part 1: Continual and DiscreteContinual Formulations of the Problems Method for Local Structural Analysis. // Applied Mechanics and Materials, 2014, Vols. 670-671, pp. 720-723.

13. Akimov P.A., Mozgaleva M.L., Aslami M., Negrozov O.A. Modified Waveletbased Multilevel Discrete-Continual Finite Element. Part 2: Reduced Formulations of the Problems in Haar Basis Method for Local Structural Analysis. // Applied Mechan- 
ics and Materials, 2014, Vols. 670-671, pp. 724-727.

14. Mozgaleva M.L., Akimov P.A. About Verification of Wavelet-Based DiscreteContinual Finite Element Method for Three-Dimensional Problems. Part 1: Structures with Constant Physical and Geometrical of Structural Analysis Parameters Along Basic Direction. // Applied Mechanics \& Materials, 2015, Vol. 709, pp. 105108.

15. Mozgaleva M.L., Akimov P.A. About Verification of Wavelet-Based DiscreteContinual Finite Element Method for Three-Dimensional Problems. Part 2: Structures with Piecewise Constant Physical and Geometrical Parameters Along Basic Direction of Structural Analysis. // Applied Mechanics \& Materials, 2015, Vol. 709, pp. 109-112.

16. Mozgaleva M.L., Akimov P.A., Kaytukov T.B. About wavelet-based multigrid numerical method of structural analysis with the use of discrete Haar basis. // International Journal for Computational Civil and Structural Engineering, 2018, Vol. 14, Issue 3, pp. 83-102.

17. Li B., Chen X. Wavelet-based numerical analysis: A review and classification. // $\mathrm{Fi}$ nite Elements in Analysis and Design, 2014, vol. 81, pp. 14-31.

18. Ko J., Kurdila A.J., Pilant M.S. A class of finite element methods based on orthonormal, compactly supported wavelets. // Comput. Mech., 1995, Vol. 16, pp. 235-244.

19. Patton R.D., Marks P.C. One-dimensional finite elements based on the Daubechies family of wavelets. // AIAAJ, 1996, Vol. 34, pp. 1696-1698.

20. Ma J.X., Xue J.J. A study of the construction and application of a Daubechies wavelet-based beam element. // Finite Elem. Anal. Des., 2003, vol. 39, pp. 965-975.

21. Chen X.F., Yang S.J., Ma J.X. The construction of wavelet finite element and its application. // Finite Elem. Anal. Des., 2004, vol. 40, pp. 541-554.
22. Li B., Cao H.R., He Z.J. The construction of one-dimensional Daubechies waveletbased finite elements for structural response analysis. // J. Vibroeng, 2011, vol. 13, pp. 729-738.

23. Jin J.M., Xue P.X., Xu Y.X., Zhu Y.L. Compactly supported non-tensor product form two-dimension wavelet finite element. // Appl. Math. Mech., 2006, Vol. 27, pp. 1673-1686.

24. Zhou Y.H., Zhou J. A modified wavelet approximation of deflections for solving PDEs of beams and square thin plates. // Finite Elements in Analysis and Design, 2008, vol. 44, pp. 773-783.

25. Mitra M., Gopalakrishnan S. Extraction of wave characteristics from wavelet-based spectral finite element formulation. // Mech. Syst. Signal Process, 2006, vol. 20, pp. 2046-2079.

26. Mitra M., Gopalakrishnan S. Wavelet Spectral element for wave propagation studies in pressure loaded axisymmetric cylinders. // J. Mech. Mater. Struct., 2007, vol. 4, pp. 753-772.

27. Mitra M., Gopalakrishnan S. Wave propagationan alysis in anisotropic plate using wavelet spectral element approach. // J. Appl. Mech., 2008, vol. 75, pp. 1-6.

28. Mitra M., Gopalakrishnan S. Waveletbased spectral finite element modelling and detection of delamination in composite beams. // Proceed. R. Soc. A, 2006, vol. 462, pp. 1721-1740.

29. Aslami M., Akimov P.A. Analytical solution for beams with multipoint boundary conditions on two-parameter elastic foundations. // Archives of Civil and Mechanical Engineering, 2016, Vol. 16, Issue 4, pp. 668-677.

30. Akimov P.A., Mozgaleva M.L. Method of Extended Domain and General Principles of Mesh Approximation for Boundary Problems of Structural Analysis. // Applied Mechanics and Materials, Vols. 580-583 (2014) pp. 2898-2902. 


\section{СПИСОК ЛИТЕРАТУРЫ}

1. Daubechies I. Orthonormal bases of compactly supported wavelets. // Commun. Pure Appl. Math., 1988, vol. 41, pp. 909996.

2. Akimov P.A., Aslami M. About Verification of Correct Wavelet-Based Approach to Local Static Analysis of Bernoulli Beam. // Applied Mechanics and Materials, 2014, Vols. 580-583, pp. 3013-3016.

3. Akimov P.A., Aslami M. Theoretical Foundations of Correct Wavelet-Based Approach to Local Static Analysis of Bernoulli Beam. // Applied Mechanics and Materials, 2014, Vols. 580-583, pp. 2924-2927.

4. Akimov P.A., Belostotsky A.M., Kaytukov T.B., Mozgaleva M.L., Aslami M. About several numerical and semianalytical methods of local structural analysis. // International Journal for Computational Civil and Structural Engineering, 2018, Vol. 14, Issue 4, pp. 59-69.

5. Akimov P.A., Mozgaleva M.L. Correct Wavelet-based Multilevel Numerical Method of Local Solution of Boundary Problems of Structural Analysis. // Applied Mechanics and Materials, 2012, Vols. 166-169, pp. 3155-3158.

6. Aslami M., Akimov P.A. Wavelet-based finite element method for multilevel local plate analysis. // Thin-Walled Structures, 2015-2016, Vol. 98, Part B, pp. 392-402.

7. Akimov P.A., Belostotsky A.M., Mozgaleva M.L., Aslami M., Negrozov O.A. Correct Multilevel Discrete-Continual Finite Element Method of Structural Analysis. // Advanced Materials Research, 2014, Vol. 1040, pp. 664-669.

8. Akimov P.A., Belostotsky A.M., Sidorov V.N., Mozgaleva M.L., Negrozov O.A. Application of discrete-continual finite element method for global and local analysis of multilevel systems. // Applied Mechanics and Materials; AIP Conference Proceedings, 2014, Vol. 1623(3), pp. 3-6.
9. Akimov P.A., Mozgaleva M.L. Correct Wavelet-based Multilevel DiscreteContinual Methods for Local Solution of Boundary Problems of Structural Analysis. // Applied Mechanics and Materials, 2013, Vols. 353-356, pp. 3224-3227.

10. Akimov P.A., Mozgaleva M.L. Waveletbased Multilevel Discrete-Continual Finite Element Method for Local Deep Beam Analysis. // Applied Mechanics and Materials, 2013, Vols. 405-408, pp. 3165-3168.

11. Akimov P.A., Mozgaleva M.L. Waveletbased Multilevel Discrete-Continual Finite Element Method for Local Plate Analysis. // Applied Mechanics and Materials, 2013, Vols. 351-352, pp. 13-16.

12. Akimov P.A., Mozgaleva M.L., Aslami M., Negrozov O.A. Modified Waveletbased Multilevel Discrete-Continual Finite Element. Part 1: Continual and DiscreteContinual Formulations of the Problems Method for Local Structural Analysis. // Applied Mechanics and Materials, 2014, Vols. 670-671, pp. 720-723.

13. Akimov P.A., Mozgaleva M.L., Aslami M., Negrozov O.A. Modified Waveletbased Multilevel Discrete-Continual Finite Element. Part 2: Reduced Formulations of the Problems in Haar Basis Method for Local Structural Analysis. // Applied Mechanics and Materials, 2014, Vols. 670-671, pp. 724-727.

14. Mozgaleva M.L., Akimov P.A. About Verification of Wavelet-Based DiscreteContinual Finite Element Method for Three-Dimensional Problems. Part 1: Structures with Constant Physical and Geometrical of Structural Analysis Parameters Along Basic Direction. // Applied Mechanics \& Materials, 2015, Vol. 709, pp. 105108.

15. Mozgaleva M.L., Akimov P.A. About Verification of Wavelet-Based DiscreteContinual Finite Element Method for Three-Dimensional Problems. Part 2: Structures with Piecewise Constant Physical and Geometrical Parameters Along Basic Direc- 
tion of Structural Analysis. // Applied Mechanics \& Materials, 2015, Vol. 709, pp. 109-112.

16. Mozgaleva M.L., Akimov P.A., Kaytukov T.B. About wavelet-based multigrid numerical method of structural analysis with the use of discrete Haar basis. // International Journal for Computational Civil and Structural Engineering, 2018, Vol. 14, Issue 3, pp. 83-102.

17. Li B., Chen $\mathbf{X}$. Wavelet-based numerical analysis: A review and classification. // Finite Elements in Analysis and Design, 2014, vol. 81, pp. 14-31.

18. Ko J., Kurdila A.J., Pilant M.S. A class of finite element methods based on orthonormal, compactly supported wavelets. // Comput. Mech., 1995, Vol. 16, pp. 235244.

19. Patton R.D., Marks P.C. One-dimensional finite elements based on the Daubechies family of wavelets. // AIAAJ, 1996, Vol. 34, pp. 1696-1698.

20. Ma J.X., Xue J.J. A study of the construction and application of a Daubechies wavelet-based beam element. // Finite Elements in Analysis and Design, 2003, vol. 39, pp. 965-975.

21. Chen X.F., Yang S.J., Ma J.X. The construction of wavelet finite element and its application. // Finite Elements in Analysis and Design, 2004, vol. 40, pp. 541-554.

22. Li B., Cao H.R., He Z.J. The construction of one-dimensional Daubechies waveletbased finite elements for structural response analysis. // J. Vibroeng, 2011, vol. 13, pp. 729-738.

23. Jin J.M., Xue P.X., Xu Y.X., Zhu Y.L. Compactly supported non-tensor product form two-dimension wavelet finite element. // Appl. Math. Mech., 2006, Vol. 27, pp. 1673-1686.

24. Zhou Y.H., Zhou J. A modified wavelet approximation of deflections for solving PDEs of beams and square thin plates. // Finite Elements in Analysis and Design, 2008, vol. 44, pp. 773-783.
25. Mitra M., Gopalakrishnan S. Extraction of wave characteristics from wavelet-based spectral finite element formulation. // Mech. Syst. Signal Process, 2006, vol. 20, pp. 2046-2079.

26. Mitra M., Gopalakrishnan S. Wavelet Spectral element for wave propagation studies in pressure loaded axisymmetric cylinders. // J. Mech. Mater. Struct., 2007, vol. 4, pp. 753-772.

27. Mitra M., Gopalakrishnan S. Wave propagationan alysis in anisotropic plate using wavelet spectral element approach. // J. Appl. Mech., 2008, vol. 75, pp. 1-6.

28. Mitra M., Gopalakrishnan S. Waveletbased spectral finite element modelling and detection of delamination in composite beams. // Proceed. R. Soc. A, 2006, vol. 462, pp. 1721-1740.

29. Aslami M., Akimov P.A. Analytical solution for beams with multipoint boundary conditions on two-parameter elastic foundations. // Archives of Civil and Mechanical Engineering, 2016, Vol. 16, Issue 4, pp. 668-677.

30. Akimov P.A., Mozgaleva M.L. Method of Extended Domain and General Principles of Mesh Approximation for Boundary Problems of Structural Analysis. // Applied Mechanics and Materials, Vols. 580-583 (2014) pp. 2898-2902.

Marina L. Mozgaleva, Dr.Sc., Professor, Department of Applied Mathematics, National Research Moscow State University of Civil Engineering, 26, Yaroslavskoe Shosse, Moscow, 129337, Russia;

phone/fax: +7(499) 183-59-94;

E-mail: marina.mozgaleva@gmail.com.

Pavel A. Akimov, Full Member of the Russian Academy of Architecture and Construction Sciences (RAACS), Professor, Dr.Sc.; Executive Scientific Secretary of Russian Academy of Architecture and Construction Sciences; Vice-Director for Science Activities, Scientific Research Center "StaDyO"; Professor of Department of Architecture and Construction, Peoples' Friendship University of Russia; Professor of Department of Structural Mechanics, Tomsk State University of Architecture and Building; 24, Ul. Bolshaya Dmitrovka, 107031, Moscow, Russia; 
phone +7(495) 625-71-63; fax: +7 (495) 650-27-31;

E-mail: akimov@raasn.ru, pavel.akimov@gmail.com.

Taymuraz B. Kaytukov, Advisor of the Russian Academy of Architecture and Construction Sciences, Associated Professor, Ph.D.; Deputy Executive Scientific Secretary of Russian Academy of Architecture and Construction Sciences; Central Institute for Research and Design of the Ministry of Construction and Housing and Communal Services of the Russian Federation; 24, U1. Bolshaya Dmitrovka, 107031, Moscow, Russia; phone +7(495) 625-81-53; fax: +7 (495) 650-27-31; E-mail: kaytukov@raasn.ru, tkaytukov@gmail.com.

Мозгалева Марина Леонидовна, доцент, доктор технических наук, профессор кафедры прикладной математики, Национальный исследовательский Московский государственный строительный университет (НИУ МГСУ), Россия, 129337, Москва, Ярославское шоссе, д.26, тел./факс: +7(499) 183-59-94,

E-mail: marina.mozgaleva@gmail.com.

Акимов Павел Алексеевич, академик Российской академии архитектуры и строительных наук (РАACH), профессор, доктор технических наук; главный ученый секретарь Российской академии архитектуры и строительных наук; заместитель генерального директора по науке ЗАО «Научно-исследовательский центр СтаДиО»; профессор Департамента архитектуры и строительства Российского университета дружбы народов; профессор кафедры строительной механики Томского государственного архитектурно-строительного университета; 107031, г. Москва, ул. Большая Дмитровка, д. 24, стр. 1; тел. +7(495) 625-71-63;

факс+7 (495) 650-27-31; E-mail: akimov@raasn.ru, pavel.akimov@gmail.com.

Кайтуков Таймураз Батразович, советник РААСН, доцент, кандидат технических наук, заместитель главного ученого секретаря Российской академии архитектуры и строительных наук; Центральный научно-исследовательский и проектный институт Министерства строительства и жилищно-коммунального хозяйства Российской Федерации; 107031, г. Москва, ул. Большая Дмитровка, д. 24, стр. 1;

тел. +7(495) 625-81-53; факс +7 (495) 650-27-31;

E-mail:kaytukov@raasn.ru,tkaytukov@gmail.com. 